\title{
PROTECTORES DE ESTRÉS LABORAL: PERCEPCIÓN DEL PERSONAL DE ENFERMERÍA Y MÉDICOS, TEMUCO, CHILE ${ }^{1}$
}

\section{PROTECTORS OF STRESS AT WORK: PERCEPTION OF A TEAM OF NURSES AND DOCTORS. TEMUCO, CHILE}

\author{
Paula R. Astudillo Díaz * \\ Ana Ma Alarcón Muñoz ${ }^{* *}$ \\ Marcela L. Lema García ${ }^{* * *}$
}

\begin{abstract}
RESUMEN
El presente estudio va en búsqueda de factores presentes en el ambiente laboral que protegen y benefician la salud en situaciones de estrés. Nuestro objetivo fue conocer los factores protectores de estrés presentes en el ambiente laboral, percibidos por el personal de salud que trabaja en centros de atención hospitalaria pública y privada de la Región de la Araucanía, Chile. Estudio cualitativo basado en teoría fundamentada de Glasser y Strauss, que contempló entrevistas en profundidad a 8 informantes claves: directivos de instituciones de salud, auxiliares de enfermería, enfermeros/as y médicos, que laboran en unidades hospitalarias críticas y no críticas, públicas y privadas de mediana y alta complejidad de la Región de la Araucanía. Fueron grabadas bajo consentimiento informado y luego transcritas para resumir los componentes que ellos percibieron. Realizado este proceso, fueron llevadas a un software para análisis textual, asignándose códigos por temas específicos y redactando comentarios. Se construyeron categorías de códigos, se compararon categorías y se establecieron relaciones entre los comentarios y las categorías para realizar análisis de conjunto. Los relatos revelan 5 temas centrales en el constructo de los protectores de estrés que se relacionan con la estructura organizacional, desarrollo del rol, liderazgo, trabajo en equipo. Los resultados pretenden ser un primer paso para develar los aspectos positivos del trabajo, útil para orientar objetivamente las futuras estrategias de promoción de salud de los trabajadores.
\end{abstract}

Palabras clave: Promoción de la salud, protectores de estrés, ambiente laboral.

\begin{abstract}
This study goes in search of the protective factors of stress in the work environment that protect and benefit health in stressful situations. Our objective was to know the stress protector factors that are present in the work environment, as perceived by the health staff who work in public and private hospitals in the Araucanía Region, Chile. It is a qualitative study based on Glasser y Strauss theory, which included in depth interviews to eight important informers: Heads of Medical Centers, nurses, aids and physicians who work in critical and non critical areas, public and private, medium and high complexity in the Araucanía Region. They were recorded under permission and transcribed to summarize what they perceived. After this process the interviews were put on a software for textual analysis, assigning codes for specific themes and then we wrote down comments. Code categories were built on and categories were compared and relations were established between the commentaries and categories in order to analyze them together. We founded five domains of stress protector factors in the
\end{abstract}

\footnotetext{
${ }^{1}$ Proyecto Evaluación de Proposiciones DIDUFRO No 120529. Universidad de la Frontera, Temuco, Chile.

* Enfermera. Magíster en Salud Pública. Profesor asociado, Departamento de Cirugía y Traumatología. Universidad de la Frontera, Temuco. Casilla 54-D, Temuco, Chile.pastudi@ufro.cl

${ }^{* *}$ Enfermera. Antropólogo. Doctorado en Antropología Médica. Profesor asociado, Departamento de Salud Pública. Universidad de la Frontera, Temuco, Chile. Teléfono: 45-325738. amalarc@ufro.cl

${ }^{* * *}$ Enfermera. Licenciada en Enfermería. Profesor asistente, Departamento de Cirugía y Traumatología. Universidad de la Frontera. Casilla 54-D, Temuco, Chile. Teléfono: 45-325760.mlema@ufro.cl
} 
organization. They were: organizational structure, clear definition of role, leadership, physical environment, and teamwork. The results pretend to be the first step to reveal the positive aspects of useful work to objectively orientate the future strategies to promote a healthy working environment for the employees.

Key words: Health promotion, stress protector, work environment.

Fecha recepción: 21/03/07 Fecha aceptación: 20/10/09

\section{INTRODUCCIÓN}

El presente estudio considera una orientación salutogénica del ambiente laboral hospitalario y va en búsqueda de factores presentes en el ambiente laboral que protegen y benefician la salud en situaciones de estrés, lo que se ajusta al plan nacional de promoción de la salud como política de estado desde 1998.

El modelo teórico para entender el estrés laboral es el modelo demanda-control-apoyo social de Karasek y Johnson, desarrollado para describir y analizar situaciones laborales en las que los estresores son crónicos y pone el acento en las características psicosociales del entorno de trabajo. Se relaciona las exigencias psicológicas a que debe dar respuesta el trabajador para desarrollar su tarea y el control que el trabajador tiene respecto a la forma, plazos y medios para realizarla. Las demandas psicológicas hacen referencia a la carga de trabajo: presión de tiempo, nivel de atención, interrupciones imprevistas; por lo tanto se circunscriben a cualquier tipo de tarea. El control o libertad de decisión se trata de la capacidad del trabajador para responder a las demandas, le permite controlar su entorno de trabajo. Hace referencia a cómo se trabaja, y tiene dos componentes: la autonomía que se refiere a la posibilidad de tomar decisiones relacionadas con su trabajo, de controlar sus propias actividades, y el desarrollo de habilidades que se refiere al grado en que el trabajo permite a la persona desarrollar sus propias capacidades, su aprendizaje, creatividad. Al modelo se agrega la dimensión de apoyo social, la que hace re- ferencia al clima social en el lugar de trabajo en relación con los compañeros y con los superiores. La función del apoyo social es la de incrementar la habilidad para hacer frente a una situación continua de estrés. El apoyo social y el control de trabajo son factores que suelen estar muy influidos por los cambios en la organización del trabajo (1).

Los factores protectores se definen como aquellas características personales o elementos del ambiente, o la percepción que se tiene sobre ellos, capaces de disminuir los efectos negativos que el proceso de estrés puede tener sobre la salud y el bienestar. Es así que ante los efectos negativos del estrés, disminuirían la vulnerabilidad y la probabilidad de enfermar (2). El NIOSH (National Institute of Occupational Health and Safety) reconoce como factores protectores en el trabajo las políticas explícitas de reconocimiento por un trabajo bien hecho, políticas de producción y de administración de recursos humanos que generen las oportunidades de desarrollo de carrera, cultura organizacional que valore al trabajador como individuo y una administración cuyas acciones y decisiones son coherentes con la declaración de valores organizacionales (3).

Las reflexiones sobre la organización de la vida cotidiana del grupo investigado que aporta su experiencia respecto a los protectores de estrés, sus características y dinámica como conjunto humano, también tienen que ver con el contexto organizacional en el que ocurre. Según describe Fonasa el año 2003, en el sistema público de salud chileno los profesionales y técnicos tienen ciertas particularidades relacionadas con la complejidad del nivel. En el secundario, existe una mayor 
participación profesional con cierto grado de diferenciación y mayor proporción de elementos de apoyo diagnóstico y terapéutico que en el nivel primario. La complejidad terciaria debe actuar como centro de referencia no sólo para la derivación de pacientes desde su propia área de influencia, sino también tiene carácter regional. Sus recursos humanos son los de la más alta especialización y los elementos de apoyo clínico diagnóstico y terapéutico, los de mayor complejidad técnica. En un equivalente al nivel terciario se establecen los hospitales del sistema privado de salud (4). En este contexto organizacional, el personal de enfermería y médicos son vulnerables en su estado de salud, dada su permanencia frente a la persona enferma $y$ su familia, la contingencia de resolver problemas emergentes como: agravamientos y accidentes, y por su trabajo frente al dolor y la muerte (5). El informe sobre "La salud en el mundo", elaborado por la OMS el año 2000, el cual está dedicado a evaluar la calidad de los sistemas de salud y cómo proceder a su mejora, señala que los recursos humanos de un sistema de salud son vitales para el buen funcionamiento y para alcanzar una buena calidad de servicio. El informe recomienda considerar variables como la satisfacción con el salario, las oportunidades para el desarrollo de la carrera profesional y las condiciones de trabajo de los profesionales (6). En nuestro país se han materializado estas recomendaciones, estructurándolas en leyes laborales. Es así como recientemente se promulgó la Ley No 19880 /2003 en relación a los funcionarios públicos y el trato laboral, considerando dentro de sus párrafos: "incentivo por desempeño", con acento sobre los ascensos y calificaciones y "carrera funcionaria por concurso".

La forma de responder de un sujeto al estrés, según Kahn el año 1970, está dada por la interpretación que da a su ambiente, influido a su vez por las variables de personalidad y relaciones interpersonales. Esta definición ha servido para múltiples estudios que invo- lucran la identificación de estresores laborales, las fuentes de estrés y la clasificación de los estresores, no obstante están centrados en qué tiene de nocivo el trabajo, más que sus características beneficiosas (7). Respecto al estrés ocupacional, los autores Lazarus y Folkman definen los esfuerzos de la persona para enfrentar el estrés laboral, llamándolo "Afrontamiento" y lo plantean como un proceso en que la persona en determinados momentos debe contar principalmente con estrategias defensivas, y en otros con estrategias que sirvan para resolver el problema, todo ello a medida que va cambiando su relación con el entorno (8). Sin embargo, surge la propuesta del médico sociólogo Aaron Antonovsky en 1984 que plantea que no sólo están presentes los recursos individuales para enfrentar una situación estresante, sino que es necesario integrarlos con los recursos significativos externos. Él los llama "recursos generalizados de resistencia" (Generalised Resistance Resources) que tienen que ver con las capacidades internas, la motivación y las posibilidades externas que nos ayudan a utilizar nuestras habilidades. A la capacidad de hacer uso de los recursos de resistencia, la describe como el Sentido de Coherencia y según relata, las personas que tienen un claro sentido del significado de sus vidas y un fuerte sistema de creencias espirituales o filosóficas soportan mejor los "desafíos de la vida" (momentos traumáticos) $(9,10)$. A esta definición se agrega la subcultura y la estructura social en la que el individuo ha crecido dando lugar a una forma determinada de ver el mundo. Entonces desde este punto de vista es posible tener en cuenta que el trabajo puede plantearse como una experiencia de vida significativa en donde la persona involucra afectos, emociones, experiencias previas, por tanto el ambiente laboral y su dinámica de desarrollo puede proporcionar al individuo nuevas experiencias de vida que aumenten o conserven su salud $(11,12)$. Actualmente los esfuerzos para promover la salud laboral se han enfocado hacia la perspectiva de "enfer- 
medad", enmarcada dentro de las categorías de enfermedades profesionales, accidentes de trabajo y problemas de salud que surgen por la exposición a riesgos ambientales como: el ruido o tóxicos $(13,14,15)$.

Creemos que los elementos que protegen y benefician la salud involucran procesos dinámicos entre el ambiente laboral y el individuo, lo que nos plantea una serie de interrogantes como: ¿a qué recursos tienen acceso las personas en su ambiente laboral para conservar su salud frente a situaciones de estrés? Si bien es cierto que un ambiente físico cómodo, acogedor y ergonométrico favorece nuestro estado de salud, no debe tomarse como un factor protector único e indiscutido ya que no está clara su relación con el estado de percepción del bienestar general (16), sólo se sabe de su relación somática. En este proceso entre individuo y organización existe un amplio campo de fenómenos que protegen la salud de los trabajadores, los cuales se vinculan a la estructura organizacional $\mathrm{y}$ a las relaciones interpersonales, es por eso que consideramos relevante estudiar, desde la percepción del auxiliar de enfermería, el profesional de enfermería y los médicos que trabajan en atención hospitalaria de mediana y alta complejidad en la Región de la Araucanía, cuáles son los elementos que reconocen como protectores de la salud en el ambiente de trabajo a nivel organizacional y grupal, frente a situaciones de estrés. Nos preguntamos entonces: ¿Cuáles son los factores protectores de estrés en el ambiente laboral, que conservan y benefician la salud, desde la percepción del personal de enfermería y médicos de la atención hospitalaria pública y privada de la Región de la Araucanía, 2005? Nuestro objetivo general es: conocer los factores protectores de estrés, presentes en el ambiente laboral, que conservan y benefician la salud desde la percepción del personal de enfermería y médicos que trabajan en atención hospitalaria pública y privada en la Región de la Araucanía. El resultado del estudio pretende que los participantes obtengan una mayor comprensión de los aspectos que conforman su realidad en el trabajo en salud, desde sus propias percepciones, como los factores protectores para su salud, además pretende ser un primer paso a enfrentar el estrés en instituciones de salud de mediana y alta complejidad, mostrando aspectos positivos del trabajo para orientar de manera objetiva las futuras estrategias regionales de promoción de la salud de los trabajadores, lo que impactará directamente a los usuarios.

\section{MATERIAL Y MÉTODO}

Metodología: Estudio cualitativo que sienta sus bases en el interaccionismo simbólico que pone énfasis en las significaciones que las personas estudiadas ponen en práctica para construir su mundo social. El análisis está dado por la Teoría Fundamentada (Grounded Theory) descrita por Glaser y Strauss (1979), que utiliza el método inductivo para descubrir y comprender procesos sociales, conceptos, proposiciones a partir de los datos, basados en la realidad laboral y no de supuestos de otras investigaciones o marcos teóricos, para obtener una descripción o explicación de los protectores de salud en situaciones de estrés, una conceptualización con bases empíricas para integrar este suceso y darle sentido. El proceso es abierto y flexible $(17,18,19)$.

Participantes: La forma de relacionarnos con los entrevistados fue a través de contactos en servicios de salud y también utilizamos la técnica de bola de nieve. Entrevistamos a 8 sujetos en los que se encuentran 1 directivo de organización pública de salud y 1 directivo del área privada, 1 auxiliar de enfermería del área pública y 1 auxiliar del área privada, 1 enfermero del área pública y 1 enfermero del área privada, 1 médico del área pública y 1 médico del área privada. Laboran en unidades tales como UTI, UCI, Recuperación anestésica, Urgencia, Servicios de hospitali- 
zado adulto de mediana y alta complejidad del área pública y privada de la Región de la Araucanía. Se caracterizan por tener entre 5 y 26 años de experiencia laboral, 5 hombres y 3 mujeres de edades entre 29 y 50 años, 5 de estado civil casados, 1 separado y 2 solteros.

Recolección de datos: Se realizan acercamientos a los sujetos en los que se explica la naturaleza de la entrevista y condiciones de su realización, asegurando el anonimato y la confidencialidad. Al momento de la entrevista en profundidad se aplica consentimiento informado. La entrevistas son grabadas en una sala privada, sin interrupciones, con una duración entre 1 y $2.5 \mathrm{~h}$. Las grabaciones una vez transcritas se borran. Nos concentramos en profundizar y comprender qué es lo que ellos visualizan como elementos protectores de estrés en su ambiente laboral. Las preguntas orientadoras fueron en dirección a explorar: "qué utiliza para protegerse del estrés", "qué elementos distingue en el ambiente laboral" considerando las características físicas, organizativas, psicosociales, "en qué se apoya para mantener su salud y el estrés no le haga daño". Esta realidad la abordamos a través del texto, el cual resume los componentes que ellos perciben. Para el término de las entrevistas se siguió el concepto de saturación teórica, considerando los criterios de propósito teórico y relevancia. El propósito teórico se refiere a los criterios iniciales del muestreo intencional cuyos rasgos se definen por la información preexistente a partir del debate teórico sobre el tema. El criterio de relevancia se refiere a ir seleccionando aquellos entrevistados que en principio nos permitan ampliar el rango de heterogeneidad, tratando de hacer emerger nuevas categorías de análisis que orienten a su vez la búsqueda de nuevos casos $(20,21)$.

Análisis de datos: Las entrevistas son transcritas y llevadas al software Atlas ti ${ }^{\circledR}$. para realizar el análisis textual inductivo, el que nos permite analizar cada categoría en conjunto, estableciendo las propiedades de cada una. La recolección de información, la codificación y el análisis se realiza simultáneamente, para ello fue necesario asignar códigos por temas específicos, redactar comentarios relacionados a los códigos, construir categorías de códigos, comparar categorías, establecer relaciones entre los comentarios y las categorías, y realizar análisis de conjunto. Se procedió a la codificación con el objetivo de acceder al universo de significados. Esta trama de códigos fue construida a partir de los ejes de la entrevista y de las dimensiones que fueron apareciendo en el proceso de lectura y redacción de comentarios de cada una de las entrevistas. Podrían señalarse dos formas de crear códigos: los códigos derivados del marco conceptual y los códigos emergentes que surgen de la lectura de las entrevistas. Se realiza triangulación del investigador en donde dos investigadores analizaron e interpretaron los datos $(20,21,22)$.

\section{RESULTADOS}

Los relatos de los participantes revelan 5 temas centrales en el constructo de los protectores de estrés que se relacionan con: 1) Puesto de trabajo; 2) Jefatura; 3) Ambiente físico; 4) Trabajo en equipo; 5) Ambiente psicosocial.

1) Respecto a los factores protectores de estrés en el Puesto de trabajo (Tabla 1), perciben que sus funciones deben estar claramente definidas y demarcadas con un perfil concreto, en donde cada uno sabe exactamente lo que le corresponde hacer, lo consideran un factor protector significativo ya que frecuentemente se enfrentan a la posibilidad de enfrentar otros turnos en los cuales su función no está definida y no saben exactamente qué es lo que les corresponde hacer. La toma de decisiones se presenta como una posibilidad entre los funcionarios, ellos están conscientes de las jerarquías y líneas de mando, pero plantean en los discursos la po- 
sibilidad de mantener cierto grado de autonomía en decidir, según criterio, eso les hace sentir que resuelven con sus propios medios los problemas. Los discursos que caracterizan la presente dimensión son: "Bien delimitada tu tarea y si hay una tarea nueva que te delimiten bien cuál es el objetivo". P1.txt - 1:138 (83:849). "Cosas clínico-administrativas que no están bien claras, las funciones, que se hace con el paciente. Las funciones de los médicos". P4.txt - 8:47 (447:454). "El hecho de tener cierta libertad de decisión o sea de resolver, intervenir y tomar decisiones, es distinto". P1.txt - 1:179 (111:116).

2) Respecto a los protectores de estrés en la Jefatura (Tabla 2), surge la idea de que asuman sus responsabilidades de guía, de directriz, que sean resolutivas, que se vean comprometidos con su tarea y con los funcionarios. Plantean como necesario aplanar las relaciones, no un jefe en la cúspide sino un jefe más participativo. Prefieren una comunicación directa, de persona a persona por sobre las cartas u oficios. Según los discursos una jefatura debe poder descansar en las capacidades de sus funcionarios. Por otra parte necesitan percibir que su jefe saldrá en su defensa, que no están solos, sentir su respaldo. Cabe destacar que en los textos se plantea una jefatura crítica, examinadora, evaluadora de la función de sus funcionarios, así como constante e invariable en las decisiones, realidad que llama la atención ya que se ve un énfasis en la autoridad y la responsabilidad, sin embargo la gestión actual da un énfasis multidimensional al liderar.

Algunos de los discursos que caracterizan esta idea central son: "Los jefes no son capaces de bajar a subordinar, no son capaces de bajar, uno no pretende que lleguen ahí a hacer box y meterse a operar con uno, en absoluto, pero sí de que haya un compromiso". P5.txt - 4:63 (374:381). "Ellas trabajan desde siempre con nosotros, conocen el trabajo que nosotros hacemos y lo valoran, hay un reconocimiento por parte de la jefatura".
P2.txt - 2:93 (99:105). "Que fiscalice, porque con una buena fiscalización se acabarían una cantidad de problemas, con una fiscalización de seis meses así dura, habrían muchos que estarían sumariados y fuera". P5.txt - 4:90 (893:898). "Te dicen tal cosa y te salen con otra cuestión, eso no estoy de acuerdo". P4.txt - 8:45 (392:395).

3) Respecto al Ambiente físico (Tabla 3), entre los elementos protectores de estrés percibidos, la posibilidad de salir del recinto, según refieren "del encierro", a un patio, a la calle o simplemente mirar el paisaje fuera del recinto de trabajo a través de una ventana es de referencia frecuente. Lo perciben como un elemento de relajo, distracción, un escenario que rompe la rutina, que da la posibilidad de pensar en otras cosas. Manifiestan no ser exigentes con los componentes estructurales en su unidad, parece no ser con la cantidad de recursos sino que la calidad de los recursos lo importante, como contar con equipamiento funcional, óptimo y con un sistema de mantención: sillas cómodas, camillas funcionales, colchones nuevos. El apoyo tecnológico lo consideran un buen soporte para el control de la evolución del paciente y en el manejo del tratamiento. Un espacio de privacidad surge como una idea más allá de ser un aporte para el descanso sino como un lugar para multiplicidad de actividades tales como: tomar decisiones de equipo, conversaciones con la familia de los pacientes, evaluación de personal. Algunos discursos que caracterizan esta dimensión son: "Tomar aire, el encierro ese de estar ahí, eso ya es estresante y el hecho de salir a donde están las ambulancias o sacar la cabeza por la ventana y tomar un poco de aire eso ya te libera un poco". P5.txt - 4:100 (108:115). "Si entráramos a un servicio donde las camas están malas, donde los baños se filtran, donde los colchones no se han podido cambiar porque no existen los recursos y los colchones están impregnados a orina, uno entra en la mañana, abre la puerta del servicio y trata de respirar antes de entrar y 
luego aperrar". P6.txt - 6:60 (449:459). "Los monitores que uno los puede programar si uno está frente a una emergencia, para que controle al paciente cada un minuto." P2.txt - 2:10 (49:57). "Tener un poco de privacidad. También tener un lugar privado donde conversar con la familia”. P8.txt - 5:87 (310:312).

4) Respecto a los protectores de estrés en el Trabajo en equipo (Tabla 4), los relatos revelan que la confianza en el conocimiento de los miembros del equipo es un elemento esencial, da la seguridad de que el compañero tiene la prudencia y el discernimiento para enfrentar situaciones críticas. Esto se encuentra muy relacionado con el sentido de pertenencia al equipo de trabajo ya que experimentan un sentimiento de ayuda y lucha ante las contingencias, saben que no están solos, los une el compromiso y la responsabilidad de cumplir los objetivos institucionales unidos. Creen en un enfrenamiento unidireccional de los problemas, en relación a las distintos roles. Necesitan de la certeza de contar con un grupo humano que responda y que piense en máximos valores, es decir, no sólo cumplir con lo que corresponde, sino que pensar siempre en hacer la tarea con excelencia, transmiten expectativas de alto rendimiento. Una percepción planteada es la de compartir el conocimiento, comunicar a los miembros del equipo el conocimiento adquirido en alguna capacitación o congreso, para dar soluciones actualizadas a los problemas y de esa manera optimizar la atención. Además, en la medida que se comparte el problema, con el conocimiento conjunto se da una mejor solución. Le dan un alto valor a la experiencia, compartir experiencias de trabajo, comunicar rutinas para soportar experiencias de estrés tiene un sentido de "resistir juntos", sirven para enfrentar situaciones similares. El sustento del grupo de trabajo ante el cansancio, las inseguridades o ante situaciones relacionadas con el cumplimiento de metas o nuevos desafíos, lo perciben como "apoyo del grupo". Uno debe pensar que una persona que está en el equipo de trabajo es un aliado, si alguien dice que está cansado es porque realmente está cansado y así apoyarse turnándose las tareas. Los discursos que caracterizan la presente dimensión son: "En la medida que uno sabe que la otra persona, es una persona experimentada, uno se apoya en los compañeros, en la enfermera, en un médico, tal vez uno se estresa cuando llega alguien nuevo". P2.txt - 2:3 (7:12). "Si uno se da cuenta que hay otra manera de hacer las cosas, un cambio en los procedimientos, en un curso, a la vuelta yo entregar todos los conocimientos". P7.txt - 7:26 (306:316). "El poder compartir experiencias en conjunto, el Dr. XX es de área ministerial, ha vivido situaciones muy similares a las mías". P4.txt - 8:9 (72:77). "Si yo comparto con otras personas esto, disminuye un poco el problema también, eso es otra forma de protegerme". P8.txt - 8:8 (79:82). "Sentirte apoyada, porque tu puedes estar súper acelerada y llena de trabajo, pero si tú sientes que estás apoyada por el grupo, es otra cosa". P7.txt - 7:9 (129:134).

5) Los protectores de estrés en el Ambiente psicosocial (Tabla 5). Ambiente psicosocial se llama a las relaciones de las personas entre sí, y las relaciones de las personas y su ambiente social al interior de la unidad de trabajo en coherencia con una visión integral de los derechos de las personas, por tanto el respeto y el lenguaje se constituyen en elementos trascendentes como protectores ante el estrés. Los trabajadores escogen un lenguaje sin gritos ni estridencias, lo que está estrechamente relacionado con el respeto al trabajo del otro. Perciben que los gritos sin medida son el reflejo de inseguridades, que el grito genera inmediatamente un ambiente "desagradable", insisten en que las situaciones adversas se conversan. Perciben que un ambiente de buen humor, agradable, entretenido hace más livianas las situaciones de estrés, lo que se une a un trato amigable y cordial, dando una mayor auten- 
ticidad y sinceridad en las relaciones . Crear situaciones de contacto, de cercanía con los otros miembros del equipo constituyen instancias en que las personas se muestran tal como son. Buscar momentos de camaradería es compartir con todos los funcionarios, es compartir vivencias fuera de las situaciones de trabajo. Compartir con todos los funcionarios fuera del horario de trabajo tiene que ver con crear escenarios que den la posibilidad de compartir, conversar otros temas, disfrutar juntos algún paseo, celebrar el día del médico, de la enfermera, el día del auxiliar. La visión de familia del equipo de trabajo, una familia con conflictos, avenencias, pero unidos, ligados sentimentalmente, denota que las vivencias laborales implican compartir experiencias críticas, solucionar problemas en conjunto, se logra una mayor cercanía con los compañeros de trabajo a los que les dan la condición de "hermanos" ya que conocen a sus familias, hijos. Años de ser miembros de un equipo de trabajo implica crecer y ser guiado, para posteriormente orientar y guiar a los trabajadores más jóvenes. Se crean vínculos afectivos fuertes, hay intimidad, en donde comparten muchas horas de vida. Los discursos que caracterizan la presente dimensión son: "Tratan de tener un ambiente agradable, te cuentan un chiste, o entre ellos se tiran bromas y uno como los va conociendo, ya va conociendo como funcionan sus vidas, también se ríe de las mismas bromas o uno los bromea". P5.txt - 4:107 (291:300). "Nosotros tenemos en nuestros murales fotos de nuestros paseos y ahí uno ve que son simpáticas las tallas. No nos faltaban situaciones de ese tipo para conversar". P4.txt - 8:60 (487:492). "Vivimos aquí de día, de noche, nos pasamos todos aquí, los nacimientos de un hijo, los matrimonios, la muerte de los papás, somos todos así con los colegas, los colegas son como hermanos". P2.txt - 2:47 (383:393). "La verdad es que es una gran familia”. P5.txt - 4:37 (301:303).

Creemos que no se puede pensar en los factores protectores de estrés presentes en el ambiente laboral sin tomar en cuenta el marco general organizacional que los propios involucrados trajeron en sus discursos. Las categorías planteadas son: objetivos organizacionales definidos y concretos, normas claras y flexibles, solución efectiva a los problemas, disponibilidad de recursos y líneas de comunicación expeditas. En relación a objetivos, para los funcionarios es significativo que sean precisos y concretos, y las normas evidentes, sus definiciones deben reflejar todo su margen de acción, esto da seguridad en el ejercicio de la labor. En los discursos aparece como un elemento de justicia que ante los problemas efectivamente existan las soluciones y no persistan ante la evidente dificultad. Que haya cambios, que la organización sea efectivamente resolutiva, que los problemas no se constituyan en rutina ya que se ven enfrentados a resolver interminablemente los mismos problemas. Desde lo anterior surge el tema de los recursos materiales y tecnológicos para dar atención eficiente y eficaz al paciente, se agrega a esto contar con recurso humano en número e idoneidad. Respecto a las líneas de comunicación, se impulsan como facilitadoras un mensaje claro y entendible. Prefieren la comunicación de persona a persona, así las recomendaciones y misiones de la organización llegarían en forma directa, rápida y expedita a los funcionarios. Los discursos que caracterizan esta visión organizacional son: "Una de las cosas es que sepamos todos qué quiere la institución o qué espera de nosotros". P7.txt 7:46 (625:630). "El hecho de estar tratando de resolver los mismos problemas administrativos, eso es lo que más te agota”. P5.txt - 4:136 (804:807). "Ir aumentando lentamente su equipamiento hasta lograr tener lo necesario que te permita seguridad laboral, yo me refiero a todos los tipos de insumos". P3.txt - 3:52 (361:367). "Yo diría que falta más atreverse a comunicarse de persona a persona porque yo creo que la gente le tiene miedo o piensan que es una nota, la derivan y es suficiente". P1.txt - 1:158 (270:272). 


\section{DISCUSIÓN Y COMENTARIO}

El concepto patogénico que caracteriza al pensamiento de estrés en el trabajo es un obstáculo a favor de la salud. En general, para resolver los problemas de estrés laboral se da a éste un criterio de "enfermedad" y no un dominio de "salud" para el trabajador, es necesario mirarlo desde una perspectiva saludable tanto para el individuo como para su grupo de trabajo, lejos de la utopía de pensar la ausencia total de estrés laboral. De aquí surgen las reflexiones en torno a que muchos trabajadores de la salud se enferman al verse expuestos a factores estresantes en el trabajo, mientras que otros siguen sanos e incluso prosperan (23). El análisis cualitativo nos permitió profundizar en los significados de las percepciones respecto a los recursos que utilizan las personas para enfrentar situaciones críticas y mantener su apreciación de bienestar. Las percepciones muestran un aparente desajuste entre las variables recomendadas por la OMS en el año 2000 , en relación al salario y oportunidades de desarrollo, ya que en la profundización de la entrevista cobra mayor importancia lo relacionado con la estructura organizacional, jefatura y relaciones humanas. Las estrategias jerárquicas planteadas para las jefaturas en los sistemas de salud son ampliamente aceptadas para manejar organizaciones, ofreciendo la promesa de eficiencia, control y rutinas predecibles. Sin embargo, se señala que la jerarquía tiende a disminuir la creatividad y el compromiso, transformando las relaciones entre trabajador y organización en una transacción puramente económica (24). Según lo planteado por los sujetos de estudio la idea es que las jefaturas logren alentar a metas de grupo, transmitan expectativas de alto rendimiento, sean capaces de crear entusiasmo intelectual y ofrecer un modelo a través de su propio comportamiento. Ahora, el apoyo social minimiza la percepción de amenaza siempre y cuando sea un aporte en información, guía, orientación y recursos a la acción. Se buscan personas que aporten o mejoren la competencia personal.

Según el modelo de Karasek y Johnson, a mayor apoyo social menor estrés y este apoyo social se consigue en un entorno de trabajo en que se fomentan las relaciones de apoyo entre los compañeros, en que el trabajador confía en que los demás compañeros le brindarán apoyo y en el que existen experiencias previas en las que se prestó ese apoyo social (1).

Como promotores de salud debemos centrarnos en pensar cómo trasformar el ambiente laboral hospitalario, de manera que este ambiente y su dinámica de desarrollo puedan proporcionar al individuo nuevas experiencias de vida, experiencias de vida significativas que conserven su salud (9). Los adultos con una vida laboral activa se enfrentan persistentemente a situaciones de estrés, por tanto las diferencias en las capacidades de cada una de las personas pueden tornarse cada vez más fuertes y positivas para la salud en la medida que logremos plantear, en la naturaleza del ambiente laboral actual, posibilidades externas, recursos protectores de salud para que los trabajadores manejen las situaciones estresantes.

Reflexionando en relación a las dificultades que surgen en el desarrollo de nuestra investigación, la primera de ellas tiene que ver con el concepto de saturación teórica. Una categoría va a estar saturada cuando seguir indagando en ella no va a agregar mayor información, sin embargo un investigador con mayor experiencia en el tema satura sus categorías antes que uno principiante, a pesar de ello se trabaja según se describe en ítem de recolección de datos. La segunda es el conflicto dado con la delimitación de las categorías y los constructos, por ejemplo se podría decir que ambiente psicosocial y trabajo en equipo no son excluyentes, sin embargo en la presente investigación el constructo "trabajo en equipo" tiene un sentido de pertenencia y el "ambiente psicosocial" a las relaciones de las personas, 
lo que nos llevó a afirmar que la categoría va a estar delimitada por la relación que guarda esa dimensión con el resto de las dimensiones en cada una de las investigaciones en particular. A pesar de estas limitaciones en la aplicación concreta de este abordaje cualitativo, el proceso es abierto y flexible, ya que se mueve en la incertidumbre(20). Consideramos que la teoría fundamentada permite que se hagan explícitos los procedimientos mediante los cuales se llega a los resultados, lo que es un gran avance en el campo de la investigación cualitativa en salud.

Ahora bien, desde la complementariedad de las metodologías de la investigación, que "en conjunto representan los dos medios fundamentales de la comunicación humana, el lenguaje verbal y el numérico" (19), un abordaje cuantitativo, nos daría la mirada para establecer categorías generales de percepción y proyectar hacia la población del estudio, además de establecer asociaciones de variables. Por tanto se proyecta sistematizar las presentes percepciones a través de la elaboración de un instrumento de recolección de datos, el cual podrá ser aplicado a funcionarios de hospitales de mediana y alta complejidad en el área pública y privada de la Región de la Araucanía, Chile.

\section{REFERENCIAS}

1. Vézina M. Las exigencias psicosociales vinculadas a la organización del trabajo. Conseiller médical en santé au travail, Institut national de santé publique du Québec. Département de médecine sociale et préventive, Université Laval, Québec, Canada. 13 marzo 2009. Hallado en: http://www.proyectoaraucaria.cl

2. Moos R H, Holahan C J. Dispositional and Contextual Perspectives on Coping: Toward an Integrative Framework. Journal of Clinical Psychology 2003; 59: 1387-1403.
3. Instituto Nacional para la Seguridad y Salud Ocupacional (NIOSH). El estrés en el trabajo. No 1999- 101. Hallado en: http://www.cdc.gov/spanish/niosh/ pubsnum-sp.html

4. Astudillo P. Estudio de clima organizacional en unidades de cuidados intensivos público y privado. Tesis de grado de Magíster en salud pública. Biblioteca de Escuela de Salud Pública, Universidad de Chile, Santiago-Chile, 1997.

5. Arenas C, Torres C, Troncoso C. Efecto moderador de la cultura organizacional en las variables de estrés laboral y tensión en el personal supervisor de ocho empresas de la novena y décima regiones. Tesis para optar al grado de Licenciado en Psicología. Universidad de la Frontera, Temuco, 2001.

6. OMS. El informe sobre "La salud en el mundo" año 2000. Hallado en : http:// www.who.int/whr/2000/es/index.html

7. Koh W L, Lim C. Stress in Social Work: Testing a Singaporean Sample, Research and Practice in Human Resource Management, 1996 4(1), 25-41.

8. Buendía J. Estrés laboral y salud. Estrés, trabajo y salud: características laborales, contexto ocupacional y diferencias individuales, 1998; (4) 79-117.

9. Antonovsky A. The estructure and properties of the sense of coherence scale. Soc Sci Med 1993, Mar; 36 (6): 725-33.

10. Larsson G, Kallenberg K. Sense of coherence, socioeconomic conditions, and health: interrelationships in a nationwide Swedish sample. European Journal of Public Health.1996;6 (3):175-80.

11. Antonovsky A. Factores saludables en el trabajo el sentido de coherencia. En R Kalimo, M Batawi y C Cooper (Comp). Los factores psicosociales en el trabajo. Ginebra. OMS.1998.

12. Olsson M, Hansson K, Lundblad A M, Cederblad M. Sense of coherence: definition and explanation. Int J Soc Welfare. 2006 ; 15: 219-229. 
13. López Mena L Campos J. Evaluación de los factores presentes en el estrés laboral. Revista de psicología de la Universidad de Chile. 2002;11(1):149-165.

14. Cruz C, Vargas L. Estrés: entenderlo es manejarlo. El estrés mirado por dentro: una perspectiva psicofisiológica. 2000: 21-35.

15. Cifuentes C, Collado T. Eficacia de un programa de gimnasia psicofísica para disminuir el nivel de estrés en los profesores de dos escuelas de educación general básica. Tesis para optar al título de Licenciado en Educación. Universidad de la Frontera, Temuco; 1995.

16. Novak P. Coping with existencial threats and the inevitability of asking for meaningfulness. Ed. Universitat ULM; 1998, octubre No 224.

17. Strauss A, Glaser B. Discovery of substantive theory: A basic strategy underlying qualitative research. Qualitative methodology: firsthand involvent with the social world. Chicago: Markham Publishing. In W J Filstead (Ed); 1979: 288304.

18. Alarcón A M, Astudillo P, Rivas E. Metodología de la investigación en enfermería. Manual. Proyecto de Desarrollo
Docente No 1620-499. Universidad de la Frontera; 2000.

19. Polit D, Hungler B. Investigación en ciencias de la salud. VI edición. México: McGraw-Hill; 2000.

20. Glaser B. Conceptualization: On theory and theorizing using grounded theory. International Journal of Qualitative Methods. 2002;1 (2). Hallado en : http:// ejournals.library.ualberta.ca/index.php/ IJQM/index

21. Strauss A, Corbin J. Bases de la investigación cualitativa: técnicas y procedimientos para desarrollar la teoría fundamentada. Facultad de Enfermería de la Universidad de Antioquia. Primera edición en español. España: Editorial Universidad de Antioquia; 2002.

22. Alarcón AM, Astudillo PR, Barrios SA, Rivas EE. Nursing leadership in Chile: a concept in transition. Nurs Sci Q. 2002;15(4):336-41.

23. Eriksson M, Lindström B. Validity of Antonovsky`s sense of coherence scale: a systematic. J. Epidemiol. Community Health. 2005; 59:460-466 .

24. Thompson A, Strickland A. Administración Estratégica. 13a edición. México: Editorial McGraw-Hill; 2003.

Tabla 1. Protectores de estrés en el Puesto de trabajo.

- Delimitación de tareas y objetivos.

- Coherencia entre rol y actividades.

- Idoneidad en los puestos de trabajo.

- Autonomía en la toma de decisiones. 
Tabla 2. Protectores de estrés en la Jefatura.

- Involucrarse en la labor de sus funcionarios.

- Confiar en las capacidades que tienen los funcionarios para realizar la labor.

- Consultar la opinión de los funcionarios.

- Respetar las decisiones tomadas en conjunto con los funcionarios.

- Ser recta y justa.

- Supervisar el trabajo de los funcionarios.

- Comunicarse de manera directa con los funcionarios.

- Valorar la contribución de funcionarios en el cumplimiento de objetivos.

Tabla 3. Protectores de estrés en el Ambiente físico.

- Posibilidad de acceso al exterior.

- Espacio que permita privacidad.

- Equipos funcionando óptimamente.

- Disponibilidad de luz natural.

- Limpio, libre de olores desagradables.

- Temperatura ambiental agradable.

Tabla 4. Protectores de estrés en el Trabajo en equipo.

- Confianza entre los miembros del equipo de trabajo.

- Sentido de pertenencia al equipo.

- Miembros comprometidos en cumplir los objetivos de la organización.

- Miembros compartan el conocimiento adquirido.

- Compartir las experiencias de trabajo vividas.

- Apoyarse en los pares ante inseguridades o desafíos.

Tabla 5. Protectores de estrés en el Ambiente psicosocial.

- Respeto al trabajo de los otros.

- Lenguaje sin gritos ni estridencias.

- Ambiente entretenido y de buen humor.

- Tener momentos de camaradería, intimidad y franqueza.

- Visión de familia del equipo de trabajo. 